\title{
Assessing sensitivity in a multidimensional space: Some problems and a definition of a general $d^{\prime}$
}

\author{
ROBIN D. THOMAS \\ Miami University, Oxford, Ohio
}

\begin{abstract}
This article provides a formal definition for a sensivity measure, $d_{g}^{\prime}$, between two multivariate stimuli. In recent attempts to assess perceptual representations using qualitative tests on response probabilities, the concept of a $d^{\prime}$ between two multidimensional stimuli has played a central role. For example, Kadlec and Townsend (1992a, 1992b) proposed several tests based on multidimensional signal detection theory that allow conclusions concerning the perceptual and/or decisional interactions of stimulus dimensions. One proposition, referred to as the diagonal $d^{\prime}$ test, relies on specific stimulus subsets of a feature-complete factorial identification task to infer perceptual separability. Also, Ashby and Townsend (1986), in a similar manner, attempted to relate perceptual independence to dimensional orthogonality in Tanner's (1956) model, which also involves $d^{\prime}$ between two multivariate signals. An analysis of the proposed $d_{g}^{\prime}$ reveals shortcomings in the diagonal $d^{\prime}$ test and also demonstrates that the assumptions behind equating perceptual independence to dimensional orthogonality are too weak. This $d_{g}^{\prime}$ can be related to a common measure of statistical distance, Mahalanobis distance, in the special case of equal covariance matrices.
\end{abstract}

A central issue that has dominated research in multidimensional perception concerns whether variations in one dimension (say, frequency of an auditory tone) affect the perceptual processing of another (e.g., amplitude). In a classic approach, various operational tasks were devised that, together, provided evidence of the separability or the integrality of stimulus dimensions (e.g., Garner, 1974). For example, if the performance of observers who were required to classify stimuli on the basis of a relevant dimension was impeded by variations of an irrelevant dimension, the stimulus dimensions were deemed integral. If no interference resulted, then the stimulus dimensions were said to be separable. Examples of integral dimensions are hue, saturation, and brightness of a color patch, as well as pitch and loudness of an auditory tone. Separable dimensions are shape of a form (circle vs. square) and brightness, as well as size of a circle and orientation of an embedded radial line.

Problems in applying these operational definitions emerged when results of one test for interactive processing conflicted with others. Some phenomena that were interpreted as revealing interactions between dimensions at the perceptual level have been shown to be simply the result of differing decision strategies employed by the participants of the study (see Maddox, 1992, for a review). In addition, stimulus integrality is not the only type of dimensional interaction that could appear. More micro-level, within-stimulus interactions are also possible (Townsend, $\mathrm{Hu}, \&$ Ashby, 1980, 1981). A promising tack to under-

Correspondence should be addressed to R. D. Thomas, Department of Psychology, Miami University, Oxford, OH 45056 (e-mail: thomasrd@ miavx1.acs.muohio.edu). standing the perception of multidimensional stimuli has grown out of unidimensional signal detection theory (SDT; Green \& Swets, 1966). Like SDT, the general recognition theory (GRT; Ashby \& Townsend, 1986) decomposes the identification process of an object into the subprocesses of perception and decision. Common to both theories is the assumption that the perceptual representation of an object is inherently noisy. Both SDT and the GRT capture this idea by representing the stimulus as a probability distribution (usually normal) defined on one (as in SDT) or multiple dimensions (as in the GRT). Facing the perceiver is the (statistical) decision problem of identifying the stimulus distribution that gave rise to the internal representation.

By adopting the rich structure of multidimensional probability theory, the GRT offered a unifying theoretical framework capturing several notions of dimensional interaction. Within the GRT, rigorous definitions of perceptual "independences" that relate observable behavioral measures, such as response probabilities and (later, e.g., Ashby \& Maddox, 1994) response times, to underlying theoretical constructs are possible. Recall that a hallmark of the GRT, as in SDT, is the distinction between perceptual and decisional processes. This allows the defining of dimensional interactions as potentially occurring at both levels. The GRT has since been refined and extended to various experimental paradigms, such as categorization (Ashby \& Gott, 1988; Maddox \& Ashby, 1993), similarity judgments (Ashby \& Perrin, 1988), preference judgments (Perrin, 1992), Garner filtering (Ashby \& Maddox, 1994; Maddox \& Ashby, 1996) and same-different classification (Thomas, 1996).

In the problem of identifying the nature of the perceptual and decisional processes underlying stimulus iden- 
tification from empirical data, different approaches can be taken. One approach is to systematically develop parametric models that differ in their assumptions and fit (i.e., estimate parameters) these models to obtained data to determine which GRT-based model yields the best overall performance relative to the number of freely varying parameters (Ashby \& Lee, 1991). From this bestfitting and most parsimonious model, inferences regarding the perceptual and decisional processes are immediate. A different methodological approach is to derive various theorems from basic axioms that relate properties holding among observable quantities, such as response probabilities or response times, to unobservable perceptual and/or decisional process assumptions. Then, empirical data could be queried for the satisfaction of those properties. This latter approach affords a possible nonparametric analysis of the data (though not always) and is usually implementable for simpler experimental designs than would be required for model fitting. Estimating parameters of complex models requires a great many degrees of freedom in the data for the model to be identifiable. This can pose practical problems for the experimenter collecting the required data, especially if the processes under study may undergo a qualitative change as a result of extended practice, as in the development of automaticity (Shiffrin \& Lightfoot, in press). For these reasons, adopting an axiomatic approach to theory evaluation may be preferred. However, the cost of taking this tack is to reduce the statistical power that is essential in identifying interesting perceptual characteristics.

Given the utility of understanding the qualitative consequences of theoretical assumptions, several investigators have taken on the task of researching the mathematical properties of the GRT (e.g., Kadlec \& Townsend, 1992a, 1992b; Thomas, 1995) and relating the results to empirical applications. For example, Kadlec and Townsend outline several theorems and propositions allowing signal detection parameters (such as $d^{\prime}$, a sensitivity measure, and $\beta$, a decision bias measure) to be used to draw conclusions regarding how the observer perceives multiple stimulus attributes. Among many other propositions, they offer a test (their Proposition 1c) to assess the perceptual separability (a kind of stimulus independence) of a pair of stimulus components referred to as the diagonal $d^{\prime}$ test. This test requires a sensitivity measure to be defined between two stimuli that physically differ on more than one dimension. In the earlier paper, Ashby and Townsend (1986) utilized a similar detection sensitivity parameter in an attempt to relate dimensional orthogonality in Tanner's (1956) detectability model (a previous concept of stimulus independence) to perceptual correlation as defined within the multidimensional GRT (see below). Both the Kadlec and Townsend and the Ashby and Townsend theoretical analyses involved the use of Euclidean geometric concepts to prove the results. This reliance on geometric constructs to establish relationships derived from probability concepts will be shown to lead to erroneous conclusions.
In the next two sections, a construction of a generalized multidimensional $d^{\prime}$ is offered. This measure represents the perceptual difference between two stimuli differing on more than one dimension that takes into account a reasonable decision strategy and the probabilist representation. Following this construction, an analysis of Kadlec and Townsend's (1992a) Proposition 1c falls short of its goal. This analysis motivates an examination of Theorems 2 and 3 of Ashby and Townsend (1986), which are also found to be incomplete. The conditions that are needed to correct Kadlec and Townsend's Proposition 1c are just those needed to adequately relate dimensional orthogonality in an early model of multidimensional SDT (Tanner, 1956) to perceptual independence defined in the GRT (Ashby \& Townsend, 1986). The relationship between the proposed multidimensional $d^{\prime}$ and existing concepts of statistical distance (e.g., Mahalanobis, 1936) is explored. Finally, the utility of this construct and other related issues appear in the General Discussion section.

\section{Multidimensional Probabilistic \\ Representations and Decision Bounds}

For exposition, suppose there are two multidimensional stimuli, $P_{1}$ and $P_{2}$, to be discriminated in a two-choice identification task. On any given trial, when a stimulus, say $P_{1}$, is presented to an observer, its internal representation is assumed to be a point, $\mathbf{x}=\left(x_{1}, x_{2}, \ldots, x_{n}\right)$, in an $n$-dimensional perceptual space in which the dimensions are assumed to correspond to the object's attributes. Across trials, due to perceptual variability, repeated presentations of $P_{1}$ give rise to a probability distribution of perceptual effects. Hence, for the overall experiment, a stimulus is modeled as a probability distribution that may be described by means, variances, and covariances. A graph of a two-dimensional distribution appears on the left in Figure 1. It is useful to view the distribution by way of an equal likelihood contour, which is obtained from a cross section of the distribution at a constant height (in the right of Figure 1). To determine what response to give to the stimulus, the observer divides the perceptual space into two mutually exclusive (and exhaustive but not necessarily connected) subsets, such that each is associated with a unique response, $R_{j}$ (" $P_{1}$ " or " $P_{2}$ "). Formally, a discrimant function, $h$, is learned, such that if $h(\mathbf{x})>0$, one response is made; whereas if $h(\mathbf{x})<0$, the other response is given. The set of points for which $h(\mathbf{x})=$ 0 is referred to as the decision bound, which serves to demarcate the response regions. A special case of interest arises when the response given depends on which stimulus' mean (i.e., perceptual prototype) is closer to the observed percept. This type of decision rule is referred to as the distance classifier (Ashby \& Gott, 1988).

If one assumes that the probability distributions for $P_{1}$ and $P_{2}$ are multivariate normal, as in standard SDT, we can define a general discrimination sensitivity as the "standardized" distance between the two perceptual means, similarly to the typical $d^{\prime}$ in SDT. The difficulty arises when we ask, "Standardized with respect to what?" 

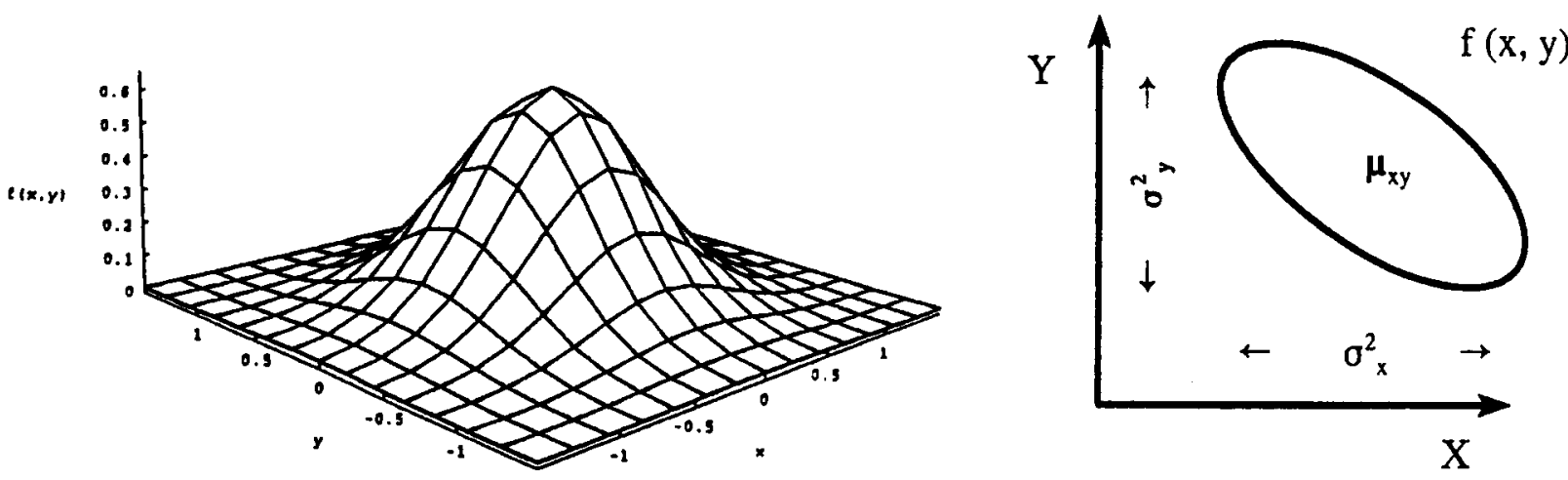

Figure 1. A bivariate Gaussian (normal) joint density function and its equal likelihood contour.

The case for two hypothetical bivariate stimuli is graphed in Figure 2. One plausible approach would be to try to measure the distance weighted by the probability densities of each stimulus distribution along a chord connecting the two perceptual means (i.e, the dotted line in Figure 2). If both two-dimensional distributions are collapsed into univariate distributions (which will be normal) over the connecting chord by integrating in the direction orthogonal to that chord (i.e., the solid line in the figure), then a distance between the means relative to the result- ing univariate standard deviations can be defined in essentially the same manner as an ordinary $d^{\prime}$. This distance, $d_{g}^{\prime}$, is a multivariate generalization of the sensitivity measure, $d^{\prime}$, from ordinary SDT.

\section{Formal Development of a}

\section{Generalized $d_{g}^{\prime}$ and the Distance Classifier}

This section formalizes the above concepts and provides a mathematical definition of the multidimensional $d_{g}^{\prime}$. Suppose the two perceptual distributions are multi-

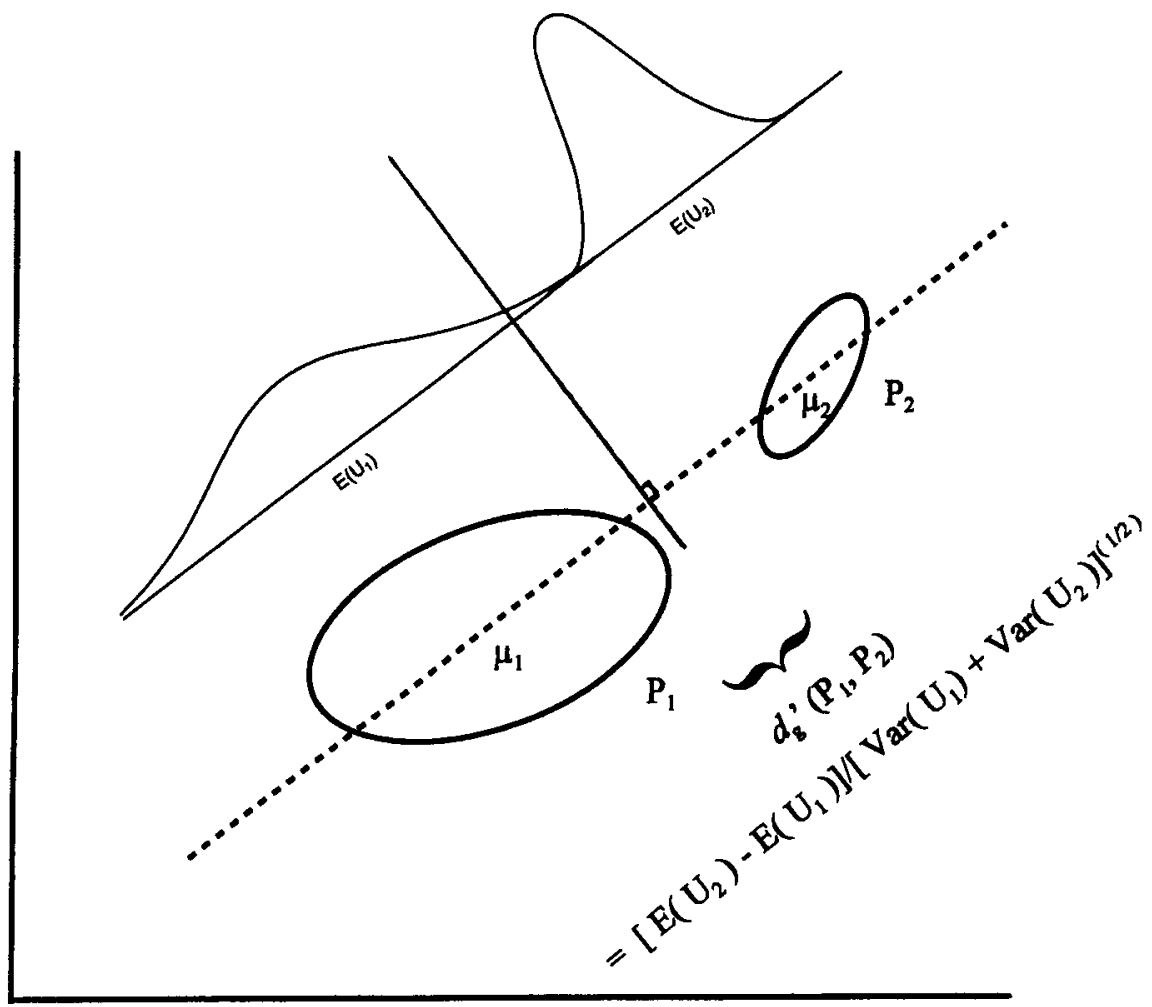

Figure 2. An illustration of the geometrical nature of the proposed generalized $d_{g}^{\prime}$ for two arbitrary distributions. Its computation assumes that a distance classifier is used to discriminate between the stimulus pair. The resulting decision criterion is a line perpendicular to the sensory axis defined by the two stimulus means (i.e., the chord connecting $\mu_{1}$ and $\mu_{2}$ ). 
variate normal with $\mathbf{X}_{1}=\left(X_{1}, X_{2}, \ldots X_{n}\right)_{1}$ denoting the random vector for $P_{1}$, and $\mathbf{X}_{2}=\left(X_{1}, X_{2}, \ldots X_{n}\right)_{2}$ for $P_{2}$. Let $\boldsymbol{\mu}_{i}$ and $\sum_{i}$ denote the mean vector and variancecovariance matrix of $P_{i}$, and let $\mathbf{v}$ be the normalized vector parallel to the chord connecting the two perceptual means-that is, $\mathbf{v}=\left(\boldsymbol{\mu}_{2}-\boldsymbol{\mu}_{1}\right) /\left\|\boldsymbol{\mu}_{2}-\boldsymbol{\mu}_{1}\right\|$, where $\|\cdot\|$ denotes the vector length. If $\boldsymbol{a}=\left(a_{1}, a_{2}\right)$ is a vector, then

$$
\|\boldsymbol{a}\|=\sqrt{a_{1}^{2}+a_{2}^{2}}
$$

Define $U_{i}$ as the univariate distribution that results from integrating $P_{i}$ along the orthogonal direction to the chord through $\boldsymbol{\mu}_{2}$ and $\boldsymbol{\mu}_{1}$. It is known from statistics that the $U_{i}$ are univariate normal random variables that are equivalent to special linear combinations of the original set of variables-that is, $U_{i}=\mathbf{v}^{T} \mathbf{X}_{i}$. Consequently, their expected values and variances are

$$
E\left(U_{i}\right)=\mathbf{v}^{T} \boldsymbol{\mu}_{i}
$$

and

$$
\operatorname{Var}\left(U_{i}\right)=\mathbf{v}^{T} \sum_{i} \mathbf{v}
$$

(e.g., Flury, 1997; Fukanaga, 1990), where $T$ denotes the vector transpose. Define a general $d_{g}^{\prime}$ as the difference between the two univariate means relative to an "average" variancel $^{l}$

$$
\begin{aligned}
d_{g}\left(P_{1}, P_{2}\right)^{\prime} & =\frac{E\left(U_{2}\right)-E\left(U_{1}\right)}{\sqrt{\operatorname{Var}\left(U_{1}\right)+\operatorname{Var}\left(U_{1}\right)}} \\
& =\frac{\mathbf{v}^{T} \boldsymbol{\mu}_{2}-\mathbf{v}^{T} \boldsymbol{\mu}_{1}}{\sqrt{\mathbf{v}^{T} \sum_{1} \mathbf{v}+\mathbf{v}^{T} \sum_{2} \mathbf{v}}} \\
& =\frac{\left(\boldsymbol{\mu}_{2}-\boldsymbol{\mu}_{1}\right)^{T}\left(\boldsymbol{\mu}_{2}-\boldsymbol{\mu}_{1}\right)}{\sqrt{\left(\boldsymbol{\mu}_{2}-\boldsymbol{\mu}_{1}\right)^{T}\left(\sum_{2}-\sum_{2}\right)\left(\boldsymbol{\mu}_{2}-\boldsymbol{\mu}_{1}\right)}} .
\end{aligned}
$$

This generalized $d_{g}^{\prime}$ is interpreted as the distance between the distribution means relative to the combined variance along the line connecting the two means, as shown in Figure 2 .

For this measure to be useful empirically, it must be computable from obtained data. In a two-choice signal detection experiment, two types of response probabilities are used in the computation of $d_{g}^{\prime}$ : the probability of responding " $P_{2}$ " given $P_{2}$ (a hit) and the probability of responding " $P_{2}$ " given $P_{1}$ (a false alarm). Once these quantities are estimated, one consults tables of hit and false-alarm rates, such as those found in Macmillan and Creelman (1991), to compute sensitivity. These response probabilities are assumed to correspond to areas underneath a standard normal distribution. Theoretical response probabilities in multidimensional detection theory are obtained by integrating the perceptual distributions (i.e., computing the volume under the distribution) over the relevant response regions demarcated by the observer's decision bounds. In order to ensure that the theoretical response probabilities from the multidimensional theory correspond to those computed from the data and used in the computation of a $d^{\prime}$, the observer's decision bound must be perpendicular to the chord connecting the two perceptual means. As stated earlier, the decision strategy yielding this type of a boundary is the distance classifier and is structurally equivalent to a prototype model in the categorization literature (Ashby \& Gott, 1988; Reed, 1972). For the $n$-dimensional case, the distance-classifier decision rule is to identify a random observation $x$ as coming from distribution $P_{1}$ if

$$
\left\|\boldsymbol{x}-\boldsymbol{\mu}_{1}\right\|^{2}-\left\|\boldsymbol{x}-\boldsymbol{\mu}_{2}\right\|^{2}<c,
$$

where $c=0$ indicates no bias, when, for example, the classes are equally likely. This decision strategy is optimal (in the sense of maximizing percent correct) only in very restricted situations (when the stimulus distributions have equal covariance matrices that are also proportional to the identity matrix). However, it may be just the kind of strategy used by novice observers in categorization tasks (Ashby \& Gott, 1988; Ashby \& Maddox, 1990; Maddox \& Ashby, 1993; but see Ashby \& Maddox, 1992). It is an open question whether this decision strategy emerges in signal detection-identification situations, although, because it is usually computationally simpler than optimal responding in a general situation, it may be the one used, particularly if the difference in accuracy obtained between optimal and distance classifying is not too large (Ripley, 1996).

\section{Characterizing Dimensional \\ Interactions within the GRT}

One application of the multidimensional representation scheme of the GRT that has proven extremely fruitful is the characterization of perceptual interactions among multiple attributes of an object. To make the discussion concrete, assume that a stimulus ensemble contains four stimuli generated by the orthogonal combination of two levels of two stimulus components, $A$ and $B$. The definitions given below generalize to more than two dimensions (Kadlec \& Townsend, 1992b), but, for exposition, this will be the case considered here. On an individual trial, one stimulus is presented for which the observer must give a unique response (i.e., identification). Denote an individual stimulus by $A_{i} B_{j}$, where $i=1$ or 2 and $j=1$ or 2 , indicating the level of the stimulus component. In a feature-complete factorial design, all four stimuli are used. The stimulus components $A$ and $B$ (e.g., frequency and amplitude of sound) are assumed to map onto two perceptual dimensions, $X$ and $Y$, respectively (e.g., pitch and loudness). A stimulus, $A_{i} B_{j}$, is represented by a bivariate random variable, $\left(\mathbf{X}_{i j}, \mathbf{Y}_{i j}\right)$, with joint density $f_{A_{i} B_{j}}(x, y)$. If the perceptual distribution is normal for stimulus $A_{i} B_{j}$, it is completely characterized by a mean vector

$$
\boldsymbol{\mu}_{A_{i} B_{j}}=\left(\begin{array}{c}
\mu_{A_{i} B_{j}}^{x} \\
\mu_{A_{i} B_{j}}^{y}
\end{array}\right)
$$

and variance-covariance matrix 


$$
\sum_{A_{i} B_{i}}=\left(\begin{array}{cc}
\sigma_{x}^{2}\left(A_{i} B_{j}\right) & \rho_{A_{i} B_{j}} \sigma_{A_{i} B_{j}}^{x} \\
\rho_{A_{i} B_{j}} \sigma_{A_{i} B_{j}}^{x} & \sigma_{y}^{2}\left(A_{i} B_{j}\right)
\end{array}\right) .
$$

A slightly different notation from that used previously is adopted for convenience of the reader in which the subscripts on the mean vector and covariance matrix will indicate which stimulus in the orthogonal set is being considered.

The observer is assumed to have divided the perceptual space into mutually exclusive and exhaustive response regions to be used for the decision process. Consequently, the identification process is simply to determine into which region a percept falls and then emit the associated response. The probability that a response " $a_{k} b_{L}$ " is given when the stimulus is $A_{i} B_{j}$ is just

$$
P\left(a_{k} b_{L} / A_{i} B_{j}\right)=\iint_{R_{a_{k} b_{L}}} f_{A_{i} B_{j}}(x, y) d x d y,
$$

where the integration is taken over the region $R_{a_{k} b_{L}}$ in the perceptual space that has been associated with the response " $a_{k} b_{L}$."

Corresponding to each joint density are two marginal densities, $g_{A_{i} B_{i}}^{x}(x)$ and $g_{A_{i} B_{i}}^{y}(y)$, associated with each perceptual dimension after the representation of the opposing dimension has been integrated out,

$$
\begin{aligned}
& g_{A_{i} B_{j}}^{x}(x)=\int_{-\infty}^{\infty} f_{A_{i} B_{j}}(x, y) d y \\
& g_{A_{i} B_{j}}^{x}(y)=\int_{-\infty}^{\infty} f_{A_{i} B_{j}}(x, y) d x .
\end{aligned}
$$

Perceptual independence (PI) of $A$ and $B$ holds within a stimulus, $A_{i} B_{j}$, if and only if the perceptual effects are statistically independent. That is, $f_{A_{i} B_{j}}(x, y)=g_{A_{i} B_{j}}^{x}(x) g_{A_{i} B_{j}}^{y}(y)$. If the densities are normal, PI is equivalent to correlation coefficient, $\rho$, equaling 0 in the stimulus. Perceptual separability (PS) of stimulus component $A$ holds if and only if, for $i=1$ and $2, g_{A_{i} B_{1}}^{x}(x)=g_{A_{i} B_{2}}^{x}(x)$. Assuming normality, PS of component $\boldsymbol{A}$ implies that the perceptual means and variances for the $X$ dimension are invariant across levels of component $B$. That is, $\mu_{A_{i} B_{1}}^{x}=\mu_{A_{i} B_{2}}^{x}$, and $\sigma_{x}^{2}\left(A_{i} B_{1}\right)=\sigma_{x}^{2}\left(A_{i} B_{2}\right)$. Similar definitions hold for PS of component $B$. If the decision regarding the level of one component, say, $A$, does not depend on the perceived value of the other component, then decisional separability (DS) holds for that component (i.e., $A$ ). For a fuller account of the various constructs and definitions emanating from the GRT, see Ashby and Townsend (1986), Kadlec and Townsend (1992a, 1992b), and Thomas (1995). Figure 3 diagrams the equal likelihood contours for a four-stimulus situation. For bivariate normal distributions, these contours are always ellipses. If $\rho=0$, then the contours are circles (if the variances are equal, i.e., $\sigma_{x}=\sigma_{y}$ ) or ellipses (in the unequal variance case) whose major and minor axes are parallel to the perceptual axes. If a perceptual correlation exists, then these axes are tilted.

\section{The Diagonal $d^{\prime}$ Test}

The diagonal $d^{\prime}$ test of Kadlec and Townsend (1992a) is a test that was proposed to reveal whether PS between stimulus components is satisfied in a data set. The test requires the following experimental trials, in addition to the feature-complete design, for its implementation. A series of blocks of trials are included that contain only $A_{1} B_{1}$ and $A_{2} B_{2}$ or only $A_{1} B_{2}$ and $A_{2} B_{1}$, in which the observer still identifies the one (of now two) presented stimulus. This latter condition is simply a two-stimulus choice task along one of the diagonals of the original stimulus ensemble square.

The empirical conditions described above allow the estimation of several discrimination sensitivities (i.e., $d$ 's). According to Kadlec and Townsend, for component $A$, there are two marginal $d$ 's, one for each level of $B$, and two diagonal $d^{\prime}$ s, one describing the discrimination sensitivity between $A_{1} B_{1}$ and $A_{2} B_{2}$ and the other describing the sensitivity between stimuli $A_{1} B_{2}$ and $A_{2} B_{1}$. The marginal $d^{\prime}$ 's are estimated from the feature-complete identification task, and their estimation requires the assumption of decision separability, whereas the diagonal $d^{\prime}$ 's are estimated from the additional "diagonal" blocks. Unfortunately, as we will see below, the authors do not provide an assumption for the decision strategy in the diagonal blocks. The authors provide the following definitions for the marginal $d^{\prime}$ s. The marginal $d^{\prime}$ for feature $A$ at the $j$ th level of feature $B$ is written as ${ }^{2}$

$$
d_{A B_{j}}^{\prime}=\frac{\mu_{A_{2} B_{j}}^{x}-\mu_{A_{1} B_{j}}^{x}}{\sqrt{\sigma_{x}^{2}\left(A_{2} B_{j}\right)+\sigma_{x}^{2}\left(A_{1} B_{j}\right)}}
$$

and for feature $B$ at the $i$ th level of $A$

$$
d_{A_{i} B}^{\prime}=-\frac{\mu_{A_{i} B_{2}}^{y}-\mu_{A_{i} B_{1}}^{y}}{\sqrt{\sigma_{y}^{2}\left(A_{i} B_{2}\right)+\sigma_{y}^{2}\left(A_{i} B_{1}\right)}} .
$$

The diagonal $d$ 's are not defined by formulas but are defined as Euclidean distances in the perceptual space between the means associated with the two presented stimuli (see Kadlec \& Towsend's, 1992a, Figure 2, p. 337). Kadlec and Townsend relate these detection parameters to PS in their Proposition 1c (assuming decisional separability for the four stimulus task):

PROPOSITION 1c of Kadlec and Townsend (1992a). PS holds for both features if and only if all three of the following conditions hold:

(i) the variances of the marginal densities, for each feature across the levels of the second feature, are equal (i.e., $\sigma_{A_{1} B_{1}}^{x}=\sigma_{A_{1} B_{2}}^{x}=\sigma_{x_{1}}, \sigma_{A_{2} B_{1}}^{x}=\sigma_{A_{2} B_{2}}^{x}=\sigma_{x_{2}}, \sigma_{A_{1} B_{1}}^{y}=\sigma_{A_{2} B_{1}}^{y}=\sigma_{y_{1}}$, and $\sigma_{A_{1} B_{2}}^{y}=\sigma_{A_{2} B_{2}}^{y}=\sigma_{y_{2}}$ );

(ii) marginal $d$ 's for both features are constant across levels of the other feature (i.e., $d_{A B_{1}}^{\prime}=d_{A_{B_{2}}}^{\prime}=d_{A}$, and $d_{A_{1} B}^{\prime}=$ $\left.d_{A_{2} B}^{\prime}=d_{B}\right)$;

(iii) $\left(d_{A}^{\prime}\right)^{2}+\left(d_{B}^{\prime}\right)^{2}=\left(d_{A B}^{\prime}\right)^{2}$, where $d_{A B}^{\prime}$ denotes the diagonal distance between the means of $f_{A_{1} B_{1}}(x, y)$ and $f_{A_{2} B_{2}}(x, y)$. We call this the Euclidean diagonal $d^{\prime}$ condition.

Proposition 1c will be shown to be in error, given our definition of a multidimensional $d_{g}^{\prime}$. The problems in the original proof of Proposition $1 \mathrm{c}$ are the use of Euclidean geometry (i.e., cosines and $d^{\prime}$ 's) for concepts defined on 


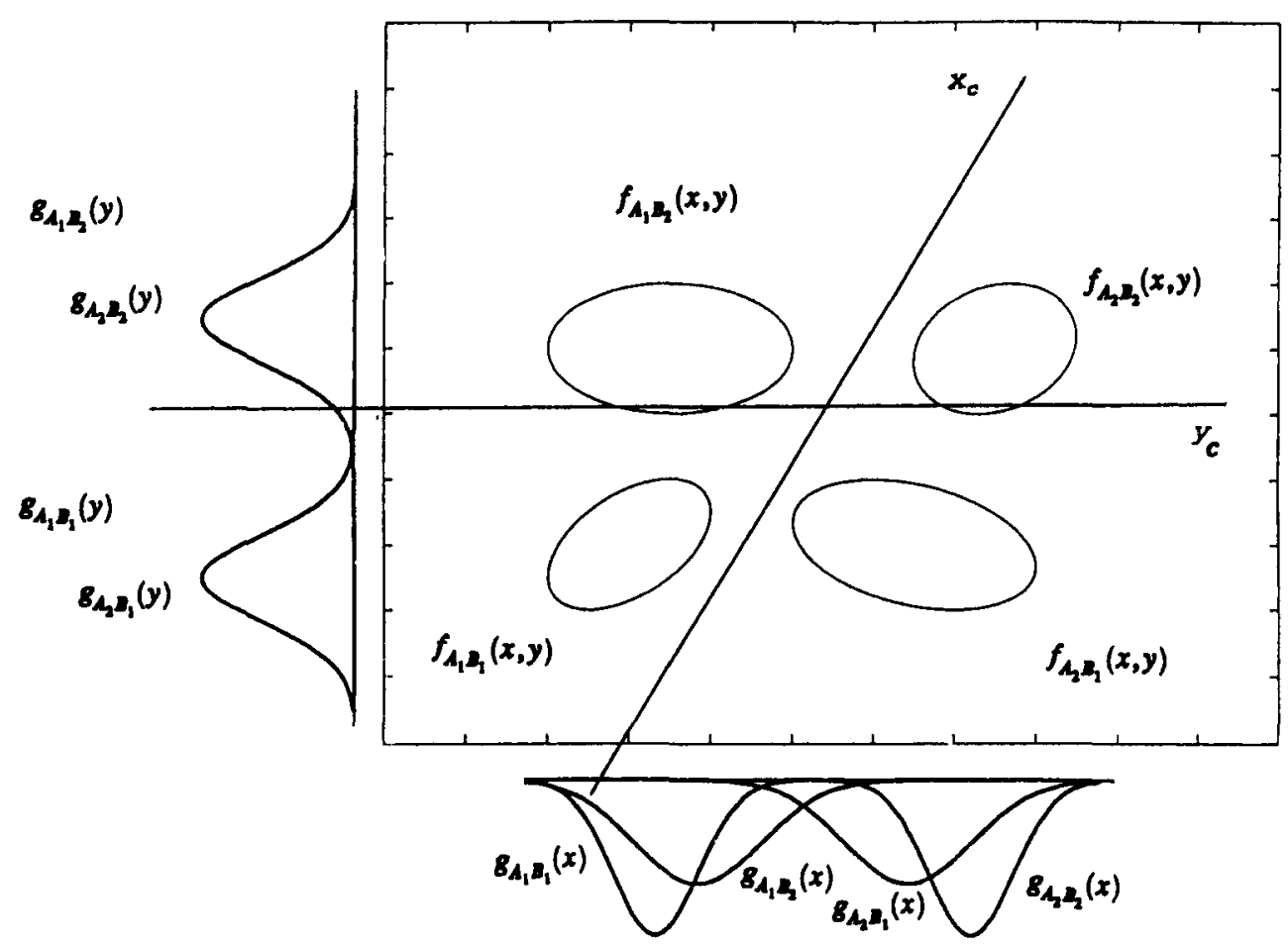

Figure 3. Equal likelihood contours of four bivariate normal densities together with their marginal density functions. Perceptual and decisional separability hold for component $B$ but not for component $A$. Also, perceptual independence is violated in all stimuli but $A_{1} B_{2}$.

probability spaces and the absence of a specified decision strategy for the diagonal trials. PS implies Conditions i and ii of the proposition. Thus, the difficulty concerns Condition iii.

Consider the discrimination between $A_{1} B_{1}$ and $A_{2} B_{2}$ with perceptual means

$$
\boldsymbol{\mu}_{A_{1} B_{1}}=\left(\begin{array}{l}
\mu_{A_{1} B_{1}}^{x} \\
\mu_{A_{1} B_{1}}^{y}
\end{array}\right)
$$

and

$$
\boldsymbol{\mu}_{A_{2} B_{2}}=\left(\begin{array}{l}
\mu_{A_{2} B_{2}}^{x} \\
\mu_{A_{2} B_{2}}^{y}
\end{array}\right) .
$$

The unit length vector in the direction of the line through $\boldsymbol{\mu}_{A_{1} B_{1}}$ and $\boldsymbol{\mu}_{A_{2} B_{2}}$ is

$$
\mathbf{v}=\frac{\left(\boldsymbol{\mu}_{A_{2} B_{2}}-\boldsymbol{\mu}_{A_{1} B_{1}}\right)}{\left\|\left(\boldsymbol{\mu}_{A_{2} B_{2}}-\boldsymbol{\mu}_{A_{1} B_{1}}\right)\right\|}=\frac{\left(\begin{array}{l}
\mu_{A_{2} B_{2}}^{x}-\mu_{A_{1} B_{1}}^{x} \\
\mu_{A_{2} B_{2}}^{y}-\mu_{A_{1} B_{1}}^{y}
\end{array}\right)}{\left\|\left(\boldsymbol{\mu}_{A_{2} B_{2}}-\boldsymbol{\mu}_{A_{1} B_{1}}\right)\right\|} .
$$

Denote by $U_{i i}$ the (univariate) random variable obtained by integration of $f_{A_{B} B}(x, y)$ in the perpendicular direction of $\mathbf{v}$ for $i=1$ and 2 . From the previous development of the multidimensional $d_{g}^{\prime}$,

$$
\begin{aligned}
E\left(U_{i i}\right)= & \mathbf{v}^{T} \boldsymbol{\mu}_{A_{i} B_{i}}\left(\mu_{A_{2} B_{2}}^{x}-\mu_{A_{1} B_{1}}^{x}\right) \\
= & \frac{\left(\mu_{A_{2} B_{2}}^{x}-\mu_{A_{1} B_{1}}^{x}\right)}{\sqrt{\left(\mu_{A_{2} B_{2}}^{x}-\mu_{A_{1} B_{1}}^{x}\right)^{2}+\left(\mu_{A_{2} B_{2}}^{y}-\mu_{A_{1} B_{1}}^{y}\right)^{2}}} \mu_{A_{i} B_{i}}^{x} \\
& +\frac{\left(\mu_{A_{2} B_{2}}^{y}-\mu_{A_{1} B_{1}}^{y}\right)^{2}}{\sqrt{\left(\mu_{A_{2} B_{2}}^{x}-\mu_{A_{1} B_{1}}^{x}\right)^{2}+\left(\mu_{i}^{y}\right.}}
\end{aligned}
$$

and

$$
\begin{aligned}
& \operatorname{Var}\left(\mathbf{U}_{i i}\right)= \\
& \mathbf{v}^{T}\left(\begin{array}{cc}
\sigma_{x}^{2}\left(A_{i} B_{i}\right) & \rho_{A_{i} B_{i}} \sigma_{x}\left(A_{i} B_{i}\right) \sigma_{y}\left(A_{i} B_{i}\right) \\
\rho_{A_{i} B_{i}} \sigma_{x}\left(A_{i} B_{i}\right) \sigma_{y}\left(A_{i} B_{i}\right) & \sigma_{y}^{2}\left(A_{i} B_{i}\right)
\end{array}\right) \mathbf{v} \\
& =\left(\frac{1}{\left\|\mu_{A_{2} B_{2}}-\mu_{A_{1} B_{1}}\right\|^{2}}\right) \sigma_{x}^{2}\left(A_{i} B_{i}\right)\left(\mu_{A_{2} B_{2}}^{x}-\mu_{A_{1} B_{1}}^{x}\right)^{2} \\
& \quad+2 \rho_{A_{i} B_{i}} \sigma_{x}\left(A_{i} B_{i}\right) \sigma_{y}\left(A_{i} B_{i}\right)\left(\mu_{A_{2} B_{2}}^{x}-\mu_{A_{1} B_{1}}^{x}\right) \\
& \\
& \quad\left(\mu_{A_{2} B_{2}}^{y}-\mu_{A_{1} B_{1}}^{y}\right)+\sigma_{y}^{2}\left(A_{i} B_{i}\right)\left(\mu_{A_{2} B_{2}}^{y}-\mu_{A_{1} B_{1}}^{y}\right)^{2} .
\end{aligned}
$$


From Equation 1, the $d^{\prime}$ between $A_{1} B_{1}$ and $A_{2} B_{2}$ is

$$
d_{A_{1} B_{1}, A_{2} B_{2}}^{\prime}=\frac{E\left(U_{22}\right)-E\left(U_{11}\right)}{\sqrt{\operatorname{Var}\left(U_{11}+\operatorname{Var}\left(U_{22}\right)\right.}} .
$$

To compute the $d^{\prime}$ between $A_{1} B_{2}$ and $A_{2} B_{1}$, one would insert the relevant perceptual means into Equations 2 and 3 with another unit length vector, $w$, in the role of $v$, which is found from

$$
\mathbf{w}=\frac{\left(\boldsymbol{\mu}_{A_{2} B_{1}}-\boldsymbol{\mu}_{A_{1} B_{2}}\right)}{\left\|\left(\boldsymbol{\mu}_{A_{2} B_{1}}-\boldsymbol{\mu}_{A_{1} B_{2}}\right)\right\|}=\frac{\left(\begin{array}{l}
\mu_{A_{2} B_{1}}^{x}-\mu_{A_{1} B_{2}}^{x} \\
\mu_{A_{2} B_{1}}^{y}-\mu_{A_{1} B_{2}}^{y}
\end{array}\right)}{\left\|\left(\boldsymbol{\mu}_{A_{2} B_{1}}-\boldsymbol{\mu}_{A_{1} B_{2}}\right)\right\|} .
$$

Figure 4 illustrates the equal likelihood contours for a very simple stimulus configuration that reveals the problem with the Kadlec and Townsend proposition. The configuration shown in Figure 4 is described by means and covariance matrices given by

$$
\begin{aligned}
& \boldsymbol{\mu}_{A_{1} B_{1}}=\left(\begin{array}{l}
0 \\
0
\end{array}\right), \sum_{A_{1} B_{1}}=\left(\begin{array}{ll}
1 & \rho \\
\rho & 1
\end{array}\right), \\
& \boldsymbol{\mu}_{A_{1} B_{2}}=\left(\begin{array}{l}
0 \\
1
\end{array}\right), \sum_{A_{1} B_{2}}=\left(\begin{array}{ll}
1 & \rho \\
\rho & 1
\end{array}\right), \\
& \boldsymbol{\mu}_{A_{2} B_{1}}=\left(\begin{array}{l}
1 \\
0
\end{array}\right), \sum_{A_{2} B_{1}}=\left(\begin{array}{ll}
1 & \rho \\
\rho & 1
\end{array}\right), \\
& \boldsymbol{\mu}_{A_{1} B_{2}}=\left(\begin{array}{l}
1 \\
1
\end{array}\right), \sum_{A_{2} B_{2}}=\left(\begin{array}{ll}
1 & \rho \\
\rho & 1
\end{array}\right),
\end{aligned}
$$

where $\rho>0$ is the correlation between the perceptual dimensions.

For the trials using only stimuli $A_{1} B_{1}$ and $A_{2} B_{2}$, from Equations 2, 3, and 4,

$$
d_{A_{1} B_{1}, A_{2} B_{2}}^{\prime}=\frac{1}{\sqrt{1+\rho}}
$$

and for trials with $A_{1} B_{2}$ and $A_{2} B_{1}$,

$$
d_{A_{1} B_{2}, A_{2} B_{1}}^{\prime}=\frac{1}{\sqrt{1-\rho}} \text {. }
$$

All marginal $d^{\prime}$ s are $1 / \sqrt{2}$. Clearly, Condition iii of the Kadlec and Townsend proposition fails, even though PS (which entails Conditions $i$ and ii) holds.

The definition of a generalized $d_{g}^{\prime}$ allows a further demonstration that PS is actually logically independent of the three conditions of Proposition 1c. The following proposition is offered.

Proposition A. When the diagonal $d^{\prime}$ is given by Equation $1,{ }^{3} \mathrm{PS}$ is logically independent of the conjunction of the three following conditions:

(i) the variances of the marginal densities, for each feature across the levels of the second feature, are equal (i.e.,
$\sigma_{A_{1} B_{1}}^{x}=\sigma_{A_{1} B_{2}}^{x}=\sigma_{x_{1}}, \sigma_{A_{2} B_{1}}^{x}=\sigma_{A_{2} B_{2}}^{x}=\sigma_{x_{2}}, \sigma_{A_{1} B_{1}}^{y}=\sigma_{A_{2} B_{1}}^{y}=\sigma_{y_{1}}$, and $\sigma_{A_{1} B_{2}}^{y}=\sigma_{A_{2} B_{2}}^{y}=\sigma_{y_{2}}$; ;

(ii) marginal $d^{\prime}$ 's for both features are constant across levels of the other feature (i.e., $d_{A B_{1}}^{\prime}=d_{A B_{2}}^{\prime}=\mathrm{d}_{A}$, and $d_{A_{1} B}^{\prime}$ $\left.=d_{A_{2} B}^{\prime}=d_{B}\right)$;

(iii) $\left(d_{A}^{\prime}\right)^{2}+\left(d_{B}^{\prime}\right)^{2}=\left(d_{A B}^{\prime}\right)^{2}$, where $d_{A B}^{\prime}$ denotes the diagonal distance between the means of $f_{A_{1} B_{1}}(x, y)$ and $f_{A_{2} B_{2}}(x, y)$. We call this the Euclidean diagonal d' condition.

That is, stimulus configurations exist such that (a) Conditions i, ii, and iii hold but PS fails, and (b) PS holds but the conjunction of Conditions i, ii, and iii fails. In particular, Condition iii fails. All proofs can be found in the Appendix. The example just given above (Figure 4) establishes (b).

Rather than a distance classifier, an alternative decision strategy for the $A_{1} B_{1}$ and $A_{2} B_{2}$ trials might be to use one of the DS decision bounds from the feature-complete task. For example, the observer may selectively attend to the $A$ component by using the criterion for the $X$ perceptual dimension to classify the two stimuli. In this case, the hit rate equals

$$
\int_{-\infty}^{\infty} \int_{x_{0}}^{\infty} f_{A_{2} B_{2}}(x, y) d x d y=\int_{x_{0}}^{\infty} g_{A_{2} B_{2}}^{x}(x) d x
$$

and the false-alarm rate equals

$$
\int_{-\infty}^{\infty} \int_{x_{0}}^{\infty} f_{A_{2} B_{1}}(x, y) d x d y=\int_{x_{0}}^{\infty} g_{A_{1} B_{1}}^{x}(x) d x .
$$

The $d^{\prime}$ estimated from these univariate normal distributions is simply the sensitivity for the $A$ component across $A_{1} B_{1}$ and $A_{2} B_{2}$. If PS holds, then this "diagonal" $d$ ' would equal both marginal $d^{\prime}$ 's for component $A$, and, hence, Condition iii of the Kadlec and Townsend proposition is false. A similar argument shows that if the observer used a DS bound for component $B$, then the diagonal $d^{\prime}$ would equal the marginal $d^{\prime}$ for $B$. Therefore, the Kadlec and Townsend proposition is not correct, even if an assumption were added that DS holds. A final possible decision strategy would be to use some kind of nonlinear decision bound (e.g., quadratic; Ashby \& Maddox, 1990, 1992; Maddox \& Ashby, 1993), which would likely be optimal in most cases. However, simply too many unknown parameters exist and too few degrees of freedom are in the data for the computation of a diagonal $d^{\prime}$ to be possible. One would need to augment the stimulus ensemble to include several levels of the components or use confidence judgments, and so on, so that different GRT models could be applied to the identification data that would allow inferences regarding the perceptual representation to be made (Ashby \& Lee, 1991). As the discussion following will establish, the Kadlec and Townsend proposition cannot be saved even if PI is assumed. A stronger condition will be required.

\section{Dimensional Orthogonality and Perceptual Independence}

Tanner (1956) developed an empirical test to determine whether a pair of tones, $S_{1}$ and $S_{2}$, differing in frequency, 


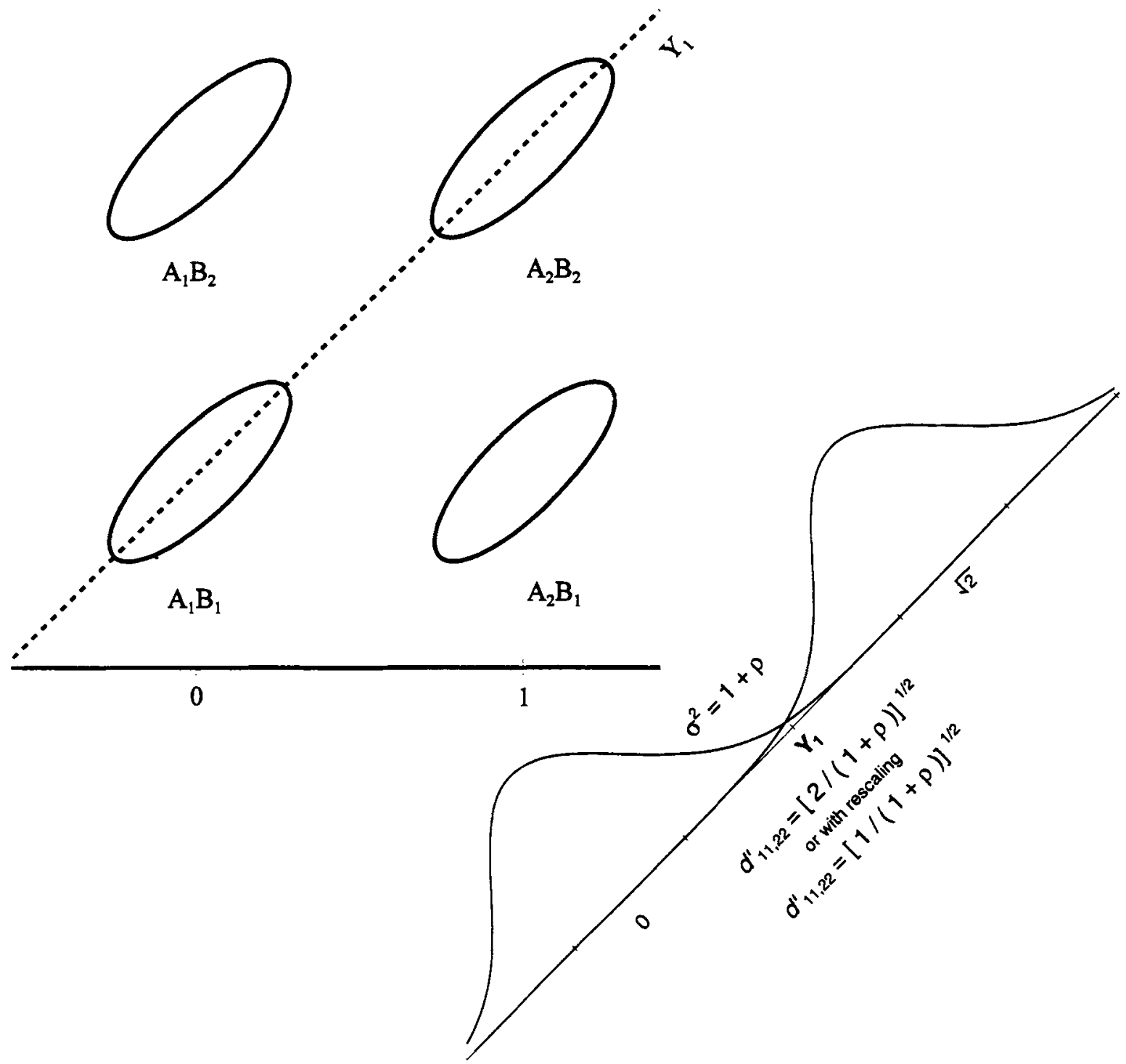

Figure 4. A stimulus configuration illustrating the failure of the diagonal $d^{\prime}$ test. The $Y_{1}$ axis corresponds to the sensory axis for stimuli $A_{1} B_{1}$ and $A_{2} B_{2}$ along which the diagonal $d^{\prime}$ is computed. The resulting diagonal $d^{\prime}$ does not satisfy Condition iii of Proposition 1c of Kadlec and Townsend, yet perceptual separability clearly holds.

were perceived independently when accompanied by white noise. His yes-no experiment consisted of three conditions: $S_{1}+$ noise trials and noise alone; $S_{2}+$ noise trials and noise alone; or $S_{1}+$ noise trials and $S_{2}+$ noise trials (i.e., a two-stimulus identification task). Never were $S_{1}$ and $S_{2}$ presented simultaneously in a trial; so any perceptual interaction had to be inferred from a comparison of detection sensitivities across conditions. Tanner adopted the bivariate normal representation for the noise and for each signal embedded in noise assuming equal variance on both dimensions and zero correlation yielding circular contours of equal likelihood (see Figure 5).

With the mean of the noise distribution at $(0,0)$, the means of the two signal distributions define the perceptual axes. The degree of perceptual dependence, according to his model, is expressed by the departure from orthogonality of the angle, $\theta$, between the perceptual dimensions defined by the signal means. To measure this, he used the Pythagorean relation

$$
\left(d_{1,2}^{\prime}\right)^{2}=\left(d_{1}^{\prime}\right)^{2}+\left(d_{2}^{\prime}\right)^{2}+2 d_{1}^{\prime} d_{2}^{\prime} \cos \theta .
$$

Note the change in subscripts on the $d^{\prime}$ 's from our earlier notation. Here, the subscripts indicate which signal, $S_{1}$ or $S_{2}$, is being considered. The $d_{1,2}^{\prime}$ is computed from the twosignal identification task and will need to assume that the decision process used is the distance classifier. The value of $\theta$ is estimated by computing the three $d^{\prime}$ s from the three experimental conditions and solving for $\theta$ in the Equation 5. This model will be referred to as the Tanner detectability model. 


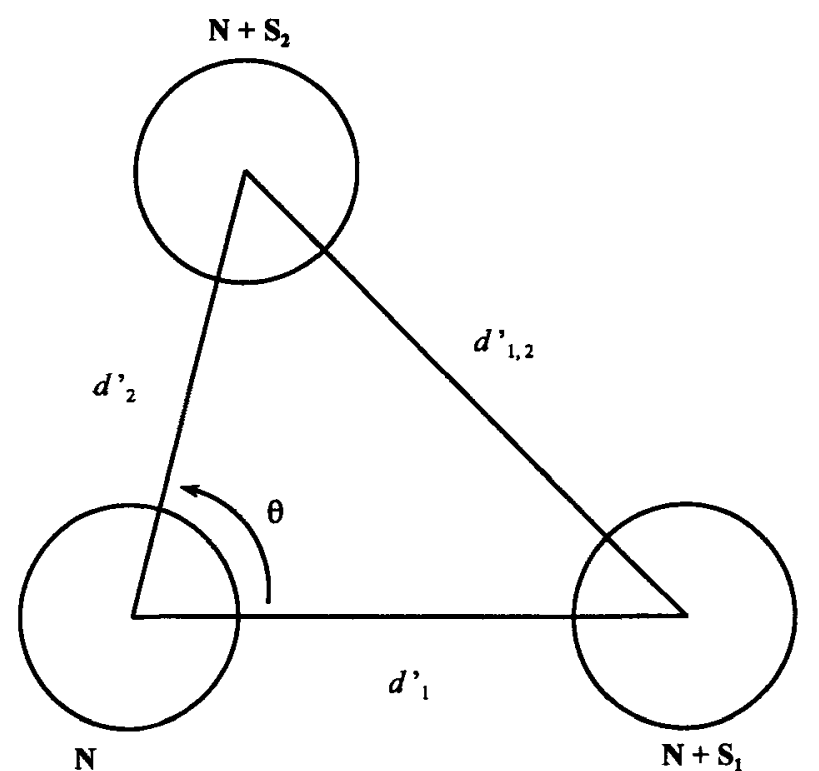

Figure 5. Contours of equal likelihood from Tanner's (1956) signal detectability model.

Ashby and Townsend (1986) consider in Theorems 2 and 3 the relationship between the Tanner detectability model and the GRT that assumes normal distributions. Theorem 2 claims that the Tanner model is a special case of the GRT, with $\cos \theta=-\rho_{x y}$ and all covariance matrices equal. In Theorem 3 , an equivalence between PI as defined in the GRT (see above) and dimensional orthogonality (i.e., $\theta=90^{\circ}$ ) in the Tanner model is apparently described. However, with the aid of the construction and analysis of the proposed multidimensional $d_{g}^{\prime}$, it will be shown that the assumptions underlying these previous theorems are too weak.

The linear transformation, L, given by Ashby and Townsend (see the proof of Theorem 2 in the Appendix of Ashby \& Townsend, 1986) renders the perceptual axis defined by $S_{2}$ to be orthogonal to the one defined by $S_{1}$,

$$
\mathbf{L}=\left(\begin{array}{cc}
1 & -\frac{\cos \theta}{\sin \theta} \\
0 & \frac{1}{\sin \theta}
\end{array}\right) .
$$

Note that the first column of $\mathbf{L}$ leaves the $S_{1}$ perceptual axis unchanged, whereas the second column rotates the $S_{2}$ axis through an angle of $90^{\circ}-\theta$. The common variance of the Tanner model is not identifiable since $d_{i}^{\prime}=\mu_{i} / \sigma$, so the equivalent GRT will be unique up to a constant scaling factor, $\sigma$.

Several aspects of this relationship need to be clarified. First, if a Tanner model adequately describes the experimental data, then the equivalent GRT exhibits stronger properties than simply equal covariance matrices. The circular contours of equal likelihood in the Tanner model (with radius unique up to the scaling factor $\sigma$ ), say, for the noise distribution, will be mapped onto ellipses defined by the following equation,

$$
x^{2}+y^{2}+2 \cos \theta x y=1 .
$$

In general, for any bivariate normal distribution, the contours of equal likelihood are described by the equation

$$
\left(\frac{x-\mu_{x}}{\sigma_{x}}\right)^{2}+\left(\frac{y-\mu_{y}}{\sigma_{y}}\right)-2 \rho_{x y} \frac{\left(x-\mu_{x}\right)\left(y-\mu_{y}\right)}{\sigma_{x} \sigma_{y}}=c^{2}
$$

for arbitrary constant $c$. With $\left(\mu_{x}, \mu_{y}\right)=(0,0)$, and letting $c^{2}=1 / \sigma^{2}$, a comparison of Equations 6 and 7 show that it must be the case that $\sigma_{x}=\sigma_{y}=\sigma$. (Recall that the Tanner contours are unique up to a scaling factor $\sigma$.) Also, for signal $S_{1}$, the mean from the Tanner model maps to

$$
\boldsymbol{\mu}_{N+S_{1}}=\left(\begin{array}{c}
\mu_{1}^{x} / \sigma \\
0
\end{array}\right)
$$

and the mean for $S_{2}$ becomes

$$
\boldsymbol{\mu}_{N+S_{2}}=\left(\begin{array}{c}
0 \\
\mu_{2}^{y} / \sigma
\end{array}\right) \text {. }
$$

Thus, Theorem 2 needs to be augmented to reflect that the true properties of the (Gaussian) GRT model that is equivalent to the Tanner model are that the means satisfy PS and the covariance matrices for the three stimuli are equal in which the variances on both dimensions are the same (i.e., $\sigma_{x}=\sigma_{y}=\sigma$ ). In examining their proof of Theorem 2, it appears that these assumptions may have been implicitly made but were not carefully stated. It is interesting to note that Equation 5 was used to relate PS to the diagonal $d^{\prime}$ in Proposition Ic of Kadlec and Townsend (1992a) with no mention of any perceptual correlation coefficient, as required by Ashby and Townsend's (1986) Theorem 2 .

Ashby and Townsend (1986) go on to state in Theorem 3 that, assuming again all covariance matrices are equal, PI (in the GRT) holds if and only if dimensional orthogonality (i.e., $\theta=90^{\circ}$ ) holds in the Tanner model. The above observation demonstrates that these conditions are also too weak. A formal restatement of the theorem is presented for reference.

THEOREM 3 of Ashby and Townsend (1986). If the covariance matrices in the general Gaussian (i.e., normal) GRT are equal, then dimensional orthogonality holds in Tanner's signal detectability model and in Tucker's (1972) general Euclidean scaling model ${ }^{4}$ if and only if components $A$ and $B$ are perceived independently.

To see that the conditions of equal covariance matrices are not enough, consider the following example. Let the three distributions $\left(N, N+S_{1}, N+S_{2}\right)$ have the Gaussian GRT representation,

$$
\boldsymbol{\mu}_{N}=\left(\begin{array}{l}
0 \\
0
\end{array}\right), \boldsymbol{\mu}_{N+S_{1}}=\left(\begin{array}{l}
0 \\
1
\end{array}\right), \boldsymbol{\mu}_{N+S_{2}}=\left(\begin{array}{l}
1 \\
0
\end{array}\right), \Sigma=\left(\begin{array}{ll}
1 & 0 \\
0 & 4
\end{array}\right) .
$$

PI holds and all covariance matrices are equal; yet when one computes the three $d$ 's (assuming a distance classifier 
is used for the discrimination between the two signals) and estimates $\theta$ from Equation 5, $\theta$ will not be $90^{\circ}$. This is because, (using the formula for $d^{\prime}$ as in Kadlec \& Townsend),

$$
\begin{aligned}
& d_{1}^{\prime}=\frac{1}{2 \sqrt{2}}, \\
& d_{2}^{\prime}=\frac{1}{\sqrt{2}},
\end{aligned}
$$

and

$$
d_{1,2}^{\prime}=\frac{2}{\sqrt{10}} \text {. }
$$

However,

$$
\left(d_{1,2}^{\prime}\right)^{2}=\frac{2}{5} \neq\left(d_{1}^{\prime}\right)^{2}+\left(d_{2}^{\prime}\right)^{2}=\frac{5}{8} .
$$

Note that neither defining $d_{1,2}^{\prime}$ as Euclidean distance nor rescaling the diagonal $d^{\prime}$ by $\sqrt{2}$ will yield the desired result. The critical aspect of this counterexample is the unequal variance for the two perceptual dimensions. The variance problem was intuited by Cohen (1997) but was incorrectly diagnosed as a failure of the distributions to be Gaussian.

To capture all of this discussion, an amended proposition is offered.

Proposition B. Dimensional orthogonality $\left(\theta=90^{\circ}\right.$ in Equation 5) holds in Tanner's (1956) detectability model if and only if all of the following conditions are met:

(i) the representation in the GRT is bivariate normal;

(ii) all covariance matrices are equal and are proportional to the identity (i.e., $\sum=\sigma^{2} I$ );

(iii) PS on the means holds (i.e., $\boldsymbol{\mu}_{N}^{x}, \boldsymbol{\mu}_{N+S_{2}}^{x}$ and $\boldsymbol{\mu}_{N+}^{y}$ $\left.s_{1}\right)$;

(iv) the decision strategy is a distance classifier.

Again, the proof can be found in the Appendix. One might take note that, under Conditions $\mathrm{i}-\mathrm{iii}$, the decision strategy that is optimal (i.e., that maximizes accuracy) is the distance classifier (Ashby \& Perrin, 1988; Fukanaga, 1990). Consequently, Condition iv could be equivalently stated as "the decision strategy is optimal."

The reader may have intuited that the conditions under which Theorem 3 of Ashby and Townsend (1986) is true are exactly those conditions under which Proposition 1c of Kadlec and Townsend (1992a) is true (with the correct definition of diagonal $d^{\prime}$ ). The equivalence between the two concepts becomes transparent when one compares the Tanner model diagramed in Figure 5 here to the Kadlec and Townsend discussion of PS and the diagonal $d^{\prime}$ condition captured by their Figure 2 (Kadlec \& Townsend, 1992a, p. 337).

\section{Statistical Measures of Distance and the Generalized $d^{\prime}$}

In statistics, there exist several concepts describing distances between multidimensional random variables (Kullback, 1968) known as measures of class separability or divergence. A complete discussion of those measures and their relationship to the proposed one is beyond the scope of this article. However, one common distance measure, referred to as Mahalanobis distance (Mahalanobis, 1936), can be readily compared to the proposed generalized $d_{g}^{\prime}$ in the special case when the two distributions are normal and have the same covariance matrix. Consider two distributions, $P_{1} \sim N\left(\boldsymbol{\mu}_{1}, \Sigma\right)$ and $P_{2} \sim N\left(\boldsymbol{\mu}_{2}, \Sigma\right)$ where $\sim$ is read "is distributed as." The Mahalanobis distance squared, $\Delta^{2}$, between them is defined as

$$
\Delta^{2}=\left(\boldsymbol{\mu}_{2}-\boldsymbol{\mu}_{1}\right)^{T} \Sigma^{-1}\left(\boldsymbol{\mu}_{2}-\boldsymbol{\mu}_{1}\right) .
$$

It can be shown that when the task is to classify samples drawn from either of the two distributions, the optimal (in the sense of maximizing percent correct) decision strategy is to use a linear discriminant function (Fukanaga, 1990). This function, label $D$, is a univariate normal random variable with mean (1/2) $\Delta^{2}$ and variance $\Delta^{2}$. Thus, Mahalanobis distance describes the divergence of the two distributions in an optimal decision situation. In the special case in which the chord connecting the two means is parallel to the linear discriminant function, $\Delta^{2}$ can be related to $d_{g}^{\prime}$

Proposition C. Assume two distributions are multivariate normal with equal covariance matrices. That is, $P_{1}$ $\sim N\left(\boldsymbol{\mu}_{1}, \Sigma\right)$ and $P_{2} \sim N\left(\boldsymbol{\mu}_{2}, \Sigma\right)$. If the chord connecting $\boldsymbol{\mu}_{1}$ and $\boldsymbol{\mu}_{2}$ is parallel to the optimal linear discriminant function, $\boldsymbol{D}$, then $\Delta^{2}=2\left(d_{g}^{\prime}\right)^{2}$.

The proof can be found in the Appendix. For a related result, see Ashby and Alfonso-Reese (1995), Theorem 4.

One consequence of this relation between $d_{g}^{\prime}$ and $\Delta^{2}$ is to observe that, in general, the distance classifier underlying $d_{g}^{\prime}$ is always going to be suboptimal except in the special case of equal covariance matrices when it is equivalent to $\Delta^{2}$ (see also Ashby \& Perrin, 1988).

\section{GENERAL DISCUSSION}

A definition of a general sensitivity parameter, $d_{g}^{\prime}$, in the multidimensional signal detection context was proposed. This is the standardized distance between the means of two multivariate normal distributions that emerges when the decision strategy is the distance classifier. A detailed analysis of the computation of this $d_{g}^{\prime}$ demonstrates that PS is logically independent of the diagonal $d^{\prime}$ condition proposed by those authors. Specifically, it was shown that, under most reasonable decision strategies, Proposition 1c of Kadlec and Townsend (1992a) does not hold without incorporating the much stronger assumptions (i.e., PS, equal covariance matrices proportional to the identity, and a distance classifier decision strategy on the diagonal trials) needed to relate dimensional orthogonality in Tanner's (1956) detectability model to PI in the GRT. The relationship between one common measure of statistical distance, Mahalanobis distance, and $d_{g}^{\prime}$ was also discussed. The need for a proposal and thorough understanding of the generalized $d_{g}^{\prime}$ is evidenced by recent empirical studies, such as those by Kingston and Macmillan (1995), Kingston, Macmillan, Dickey, and Thorburn (1997), Cohen (1997), and Kubovy, Cohen, and Hollier (1999). In each of these 
papers, the amount of perceptual interaction, specifically perceptual integrality, between two stimulus dimensions was inferred from the relation between various $d$ 's in a manner similar to the diagonal $d^{\prime}$ condition of Kadlec and Townsend (1992a). Fortunately, in all cases, the authors assumed the perceptual correlation to be 0 , which, by virtue of Proposition B in the present article, allows the desired comparisons to be made.

One interesting consequence of defining $d_{g}^{\prime}$ in this way is to note that the marginal $d^{\prime}$ for one component, say, between $A_{1} B_{1}$ and $A_{2} B_{1}$ as defined in Kadlec and Townsend (1992a) and above, will not, in general, be equal to the $d_{g}^{\prime}$ between the same two stimuli. These quantities will be equal only in the case in which the chord connecting the two perceptual means is parallel to the perceptual dimension associated with that component. This insight can be used to help alleviate the difficulties in detecting true PS, which Proposition 1c of Kadlec and Townsend (1992a) intended to do. The Kadlec and Townsend proposition was an attempt to empirically discriminate between a parallelogram (nonrectangular) perceptual configuration (see their Figure 2, p. 337) and a rectangular one given by PS. Both configurations would yield equal marginal $d$ 's for both components assuming DS. In the featurecomplete factorial design, a decisionally separable strategy might be the most likely to be used by an observer, due to the structure of the stimulus set, instructions, and cognitive demands. However, if one included only trials in which two stimuli that shared a value on one component, say, $B$, in a series of blocks, then a more accurate decision strategy might be a distance classifier. The $d^{\prime}$ estimated from these trials then would equal $d_{g}^{\prime}$ proposed here. If the observed $d_{g}^{\prime}$ equaled the marginal $d^{\prime}$ for component $A$ from the feature-complete factorial trials, then evidence for PS of component $B$ would be obtained, at least for the perceptual means. A similar suggestion to augment the feature-complete factorial design with trials of this type was made by Thomas (1995) in clarifying a test for PI proposed by Ashby and Townsend (1986, Theorem 4). Other reasons to add such trials includes the ability to use response time distributions to convergently assess separability (Ashby \& Maddox, 1994).

The definition and use of the multidimensional $d^{\prime}$ is in the spirit of the axiomatic tradition of model evaluation. The virtues of the axiomatic approach over a model fitting approach include the former's applicability to empirical designs yielding fewer data points. To fit a full GRT model to identification data requires the estimation of a great many parameters (i.e., all the means, variances, correlations, and, in general, the type and location of the decision boundaries). In many cases, this type of application may make the theory difficult to test due to fitting noise in the data rather than capture genuine perceptual trends, a situation referred to as overfitting or overparameterization (McKinley \& Nosofsky, 1996; Nosofsky \& Smith, 1992). Experimental designs that generate suffi- cient amounts of data for this purpose often require numerous trials, placing an undue burden on the participant. The resulting extensive practice may lead to a fundamental change in the nature of the perceptual representation (Shiffrin \& Lightfoot, in press), obscuring the original representation. Consequently, simpler and shorter experiments may be preferred. On the other hand, the model fitting approach has been shown to be more powerful, in the statistical sense, than testing broad properties in the data (Kadlec, 1993), such as the equivalence of marginal $d$ 's. High statistical power is essential before conclusions of independence and/or separability can be drawn-conclusions that are essentially acceptances of the null hypothesis (Loftus, 1996). The modeling approach also allows a full specification of the observer's decision process, which can become quite complex (Maddox \& Ashby, 1993). Both perceptual and decisional strategies are involved in the identification process, so neither can be ignored when the one or the other is of interest.

In summary, a generalized measure of multidimensional perceptual distance, $d_{g}^{\prime}$, was proposed on the basis of the decision strategy of classifying an observation as from the stimulus with the nearest perceptual mean. A full analysis of the relationship between PI, PS, and signal detection parameters indicates that previous propositions in Kadlec and Townsend (1992a, 1992b) and Ashby and Townsend (1986) are incomplete. The present discussion highlights the importance of the decision process and the inapplicability of geometric concepts to the computation of detection sensitivities.

\section{REFERENCES}

Ashby, F. G., \& Alfonso-ReEse, L. A. (1995). Categorization as probability density estimation. Journal of Mathematical Psychology, 39, 216-233

AshBy, F. G., \& Gort, R. (1988). Decision rules in the perception and categorization of multidimensional stimuli. Journal of Experimental Psychology: Learning, Memory, \& Cognition, 14, 33-53.

AshBY, F. G., \& LEE, W. W. (1991). Prediction similarity and categorization from identification. Journal of Experimental Psychology: General, 120, 150-172.

AshBY, F. G., \& MADDox, W. T. (1990). Integrating information from separable psychological dimensions. Journal of Experimental Psy. chology: Human Perception \& Performance, 16, 598-612.

Ashby, F. G., \& Maddox, W. T. (1992). Complex decision rules in categorization: Contrasting novice and experienced performance. Journal of Experimental Psychology: Human Perception \& Performance, 18, 50-71.

Ashby, F. G., \& MADDox, W. T. (1994). A response time theory of separability. Journal of Mathematical Psychology, 38, 423-466.

Ashby, F. G., \& Perrin, N. A. (1988). Toward a unified theory of similarity and recognition. Psychological Review, 95, 124-150.

Ashby, F. G., \& TownsEnd, J. T. (1986). Varieties of perceptual independence. Psychological Review, 93, 154-179.

CoHen, D. J. (1997). Visual detection and perceptual independence: Assessing color and form. Perception \& Psychophysics, 59, 623-635.

FlURY, B. (1997). A first course in multivariate statistics. New York: Springer-Verlag.

FUKANAGA, K. (1990). Introduction to statistical pattern recognition (2nd ed.). Boston: Academic Press. 
GARNER, W. R. (1974). The processing of information and structure New York: Wiley

Green, D. M., \& Swets, J. A. (1966). Signal detection theory and psychophysics. New York: Wiley.

KADLEC, H. (1993, August). Type I error and power of a hierarchical general recognition model: Testing and signal detection analyses of dimensional interactions. Paper presented at the Twenty-Sixth Annual Meeting of the Society for Mathematical Psychology, Norman, OK.

KADLEC, H., \& TOWNSEND, J. T. (1992a). Implications of marginal and conditional detection parameters for the separabilities and independence of perceptual dimensions. Journal of Mathematical Psychology, 36, 325-374

KadLeC, H., \& Townsend, J. T. (1992b). Signal detection analyses of dimensional interactions. In F. G. Ashby (Ed.), Multidimensional mod els of perception and cognition (pp. 181-227). Hillsdale, NJ: Erlbaum.

KinGSTON, J., \& MACMILLAN, N. A. (1995). Integrality of nasalization and F-sub- 1 in vowels in isolation and before oral and nasal consonants: A detection-theoretic application of the Garner paradigm. Journal of the Acoustical Society of America, 97, 1261-1285.

Kingston, J., Macmillan, N. A., Dickey, L. W., \& Thorburn, R. (1997). Integrality in the perception of tongue root position and voice quality in vowels. Journal of the Acoustical Society of America, 101 1696-1709.

Kubovy, M., Cohen, D., \& Hollier, J. (1999). Feature integration that routinely occurs without focal attention. Psychonomic Bulletin \& Review, 6, 183-203.

Kullback, S. (1968). Information theory and statistics. New York Dover.

LofTus, G. R. (1996). Psychology will be a much better science when we change the way we analyze data. Current Directions in Psychological Science, 5, 161-171.

Macmillan, N. A., \& Creelman, C. D. (1991). Detection theory: A user's guide. Cambridge: Cambridge University Press

Madbox, W. T. (1992). Perceptual and decisional separability. In F. G. Ashby (Ed.), Multidimensional models of perception and cognition (pp. 147-180). Hillsdale, NJ: Erlbaum.

MADDox, W. T., \& AshBy, F. G. (1993). Comparing decision bound and exemplar models of categorization. Perception \& Psychophysics, $\mathbf{5 3}$ 49-70.

Maddox, W. T., \& Ashby, F. G. (1996), Perceptual separability, decisional separability, and the identification-speeded classificatioin relationship. Journal of Experimental Psychology: Human Perception \& Performance, 22, 795-817.

Mahalanobis, P. C. (1936). On the generalized distance in statistics Proceedings of the National Institute for Science in India, 12, 49-55.

McKinley, S. C., \& Nosofsky, R. M. (1996). Investigations of exemplar and decision bound models in large, ill-defined category structures. Journal of Experimental Psychology: Human Perception \& Performance, 21, 128-148.

Nosofsky, R. M., \& SMITH, J. E. K. (1992). Similarity, identification, and categorization: Comment on Ashby and Lee (1991). Journal of Experimental Psychology: General, 121, 237- 245.

PERRIN, N. A. (1992). Uniting similarity, identification, and preference:
General recognition theory. In F. G. Ashby (Ed.), Multidimensional mod els of perception and cognition (pp. 123-146). Hillsdale, NJ: Erlbaum.

REED, S. K. (1972). Pattern recognition and categorization. Cognitive Psychology, 3, 382-407.

RIPLEY, B. D. (1996). Pattern recognition and neural networks. New York: Cambridge University Press.

Shiffrin, R. M., \& LightFoot, N. (in press). Perceptual learning of alphanumeric-like characters. In R. L. Goldstone, P. Schyns, \& D. Medin (Eds.), Mechanisms of perceptual learning. New York: Academic Press.

TANNER, W. P. (1956). Theory of recognition. Journal of the Acoustical Society of America, 28, 882-888.

Thomas, R. D. (1995). Gaussian general recognition theory and perceptual independence. Psychological Review, 102, 192-200.

Thomas, R. D. (1996). Separability and independence of dimensions within the same-different judgment task. Journal of Mathematical Psychology, 4, 318-341.

Townsend, J. T., Hu, G. G., \& AsHby, F. G. (1980). A test of visual feature sampling independence with orthogonal straight lines. Bulletin of the Psychonomic Society, 15, 163-166.

Townsend, J. T., Hu, G. G., \& Ashby, F. G. (1981). Perceptual sampling of orthogonal straight line features. Psychological Research, 43, 259-275

TuCker, L. R. (1972). The relations between multidimensional scaling and three-mode factor analysis. Psychometrika, 37, 3-27.

\section{NOTES}

1. To be consistent with ordinary SDT, the formula would also need to divide the quantity under the square root sign in the denominator by 2. However, when Kadlec and Townsend defined $d^{\prime}$ along an individual dimension for distributions with potentially unequal variances, for unknown reasons, the 2 was omitted. Thus, the present definition is of fered to be consistent with their definition.

2. The definitions for the marginal $d$ 's given by Kadlec and Townsend are also not estimable from the data in a $2 \times 2$ featurecomplete factorial design. In the estimation of $d^{\prime}$ in this case an assumption of equal variances for the two stimuli in question would have to be made as in standard SDT.

3. One objection to the above analysis is that in Proposition lc of Kadlec and Townsend, the diagonal $d^{\prime}$ is defined as the Euclidean distance between the means for the stimuli occupying the corners of the configuration. Perhaps the proposition would be true if the diagonal $d^{\prime}$ were defined in such a way. Unfortunately, the proposition is not correct even with Euclidean definition. Consider the stimulus configuration shown in Figure 4. Now, let $\rho=0$. The Euclidean distance between the means for $A_{1} B_{1}$ and $A_{2} B_{2}$ is $\sqrt{2}$, yet the two marginal $d$ 's are both equal to $1 / \sqrt{2}$. A check of Condition iii shows that the diagonal $d^{\prime}$ condition fails, even though the stimulus condition enjoys perceptual separability and independence. The difficulty is that the distance needs to be weighted by the probability functions.

4. Ashby and Townsend (1986) do not discuss the Tucker scaling model but refer the reader to a later published paper, Ashby and Perrin (1988) for this model's relationship to the GRT 


\section{APPENDIX}

\section{Proof of Proposition A}

ProOF a. Let the four stimulus distributions have the following means and covariance matrices:

$$
\begin{aligned}
& \boldsymbol{\mu}_{A_{1} B_{1}}=\left(\begin{array}{l}
0 \\
0
\end{array}\right), \sum_{A_{1} B_{1}}=\left(\begin{array}{cc}
1 & \rho_{11} \\
\rho_{11} & 1
\end{array}\right), \boldsymbol{\mu}_{A_{1} B_{2}}=\left(\begin{array}{l}
x_{1} \\
y_{2}
\end{array}\right), \sum_{A_{1} B_{2}}=\left(\begin{array}{cc}
1 & \rho_{12} \\
\rho_{12} & 1
\end{array}\right) \\
& \boldsymbol{\mu}_{A_{2} B_{1}}=\left(\begin{array}{l}
x_{2} \\
y_{1}
\end{array}\right), \Sigma_{A_{2} B_{2}}=\left(\begin{array}{cc}
1 & \rho_{21} \\
\rho_{21} & 1
\end{array}\right), \boldsymbol{\mu}_{A_{2} B_{2}}=\left(\begin{array}{l}
x_{1}+x_{2} \\
y_{1}+y_{2}
\end{array}\right), \sum_{A_{2} B_{2}}=\left(\begin{array}{cc}
1 & \rho_{22} \\
\rho_{22} & 1
\end{array}\right) .
\end{aligned}
$$

Clearly, Condition i holds with all variances equal to 1 , and Condition ii holds with all marginal $d$ 's on the $X$ dimension equal to $x_{2} / \sqrt{2}$ and the marginal $d$ 's on the $Y$ dimension equal to $y_{2} / \sqrt{2}$, with the Kadlec and Townsend definition of marginal $d^{\prime}$. Note, however, because the means on each dimension are not invariant across levels of the other component, perceptual separability fails. All that needs to be shown is that the diagonal $d^{\prime}$ condition is nonetheless satisfied.

Consider stimuli $A_{1} B_{1}$ and $A_{2} B_{2}$. From Equation 2, $E\left(\mathbf{U}_{11}\right)=0$,

$$
\begin{gathered}
E\left(U_{22}\right)=\sqrt{\left(x_{1}+x_{2}\right)^{2}+\left(y_{1}+y_{2}\right)^{2}}, \\
\operatorname{Var}\left(U_{11}\right)=\frac{\left(x_{1}+x_{2}\right)^{2}+2 \rho_{11}\left(x_{1}+x_{2}\right)\left(y_{1}+y_{2}\right)+\left(y_{1}+y_{2}\right)^{2}}{\left(x_{1}+x_{2}\right)^{2}+\left(y_{1}+y_{2}\right)^{2}},
\end{gathered}
$$

and

$$
\operatorname{Var}\left(U_{22}\right)=\frac{\left(x_{1}+x_{2}\right)^{2}+2 \rho_{22}\left(x_{1}+x_{2}\right)\left(y_{1}+y_{2}\right)+\left(y_{1}+y_{2}\right)^{2}}{\left(x_{1}+x_{2}\right)^{2}+\left(y_{1}+y_{2}\right)^{2}} .
$$

Substituting these quantities into Equation 4 and simplifying,

$$
d_{A_{1} B_{1}, A_{2} B_{2}}^{\prime}=\frac{\left(x_{1}+x_{2}\right)^{2}+\left(y_{1}+y_{2}\right)^{2}}{\sqrt{2\left(x_{1}+x_{2}\right)^{2}+\left(2 \rho_{11}+2 \rho_{22}\right)\left(x_{1}+x_{2}\right)\left(y_{1}+y_{2}\right)+2\left(y_{1}+y_{2}\right)^{2}}} .
$$

For stimuli $A_{1} B_{2}$ and $A_{2} B_{1}$,

$$
\begin{gathered}
E\left(U_{12}\right)=\frac{x_{1}\left(x_{2}-x_{1}\right)+y_{2}\left(y_{1}-y_{2}\right)}{\sqrt{\left(x_{2}-x_{1}\right)^{2}+\left(y_{1}-y_{2}\right)^{2}}}, \\
E\left(U_{21}\right)=\frac{x_{2}\left(x_{2}-x_{1}\right)+y_{1}\left(y_{1}-y_{2}\right)}{\sqrt{\left(x_{2}-x_{1}\right)^{2}+\left(y_{1}-y_{2}\right)^{2}}}, \\
\operatorname{Var}\left(U_{12}\right)=\frac{\left(x_{2}-x_{1}\right)^{2}+2 \rho_{12}\left(x_{2}-x_{1}\right)\left(y_{1}-y_{2}\right)+\left(y_{1}-y_{2}\right)^{2}}{\left(x_{2}-x_{1}\right)^{2}+\left(y_{1}-y_{2}\right)^{2}},
\end{gathered}
$$

and

Again, from Equation 4,

$$
\operatorname{Var}\left(U_{21}\right)=\frac{\left(x_{2}-x_{1}\right)^{2}+2 \rho_{21}\left(x_{2}-x_{1}\right)\left(y_{1}-y_{2}\right)+\left(y_{1}-y_{2}\right)^{2}}{\left(x_{2}-x_{1}\right)^{2}+\left(y_{1}-y_{2}\right)^{2}} .
$$

$$
d_{A_{1} B_{2}, A_{2} B_{1}}^{\prime}=\frac{\left(x_{2}-x_{1}\right)^{2}+\left(y_{1}-y_{2}\right)^{2}}{\sqrt{2\left(x_{2}-x_{1}\right)^{2}+\left(2 \rho_{12}+2 \rho_{21}\right)\left(x_{2}-x_{1}\right)\left(y_{1}-y_{2}\right)+2\left(y_{1}-y_{2}\right)^{2}}} .
$$

For the diagonal $d^{\prime}$ condition (i.e., Condition iii of the Kadlec and Townsend proposition) to be satisfied, any $x_{1}, x_{2}, y_{1}, y_{2}, \rho_{11}, \rho_{12}, \rho_{21}$, and $\rho_{22}$ that satisfy three simultaneous equations

$$
\begin{aligned}
& \frac{\left(x_{2}-x_{1}\right)^{2}+\left(y_{1}-y_{2}\right)^{2}}{\sqrt{2\left(x_{2}-x_{1}\right)^{2}+2\left(\rho_{12}+\rho_{21}\right)\left(x_{2}-x_{1}\right)\left(y_{1}-y_{2}\right)+2\left(y_{1}-y_{2}\right)^{2}}} \\
& =\frac{\left(x_{1}+x_{2}\right)^{2}+\left(y_{1}+y_{2}\right)^{2}}{\sqrt{2\left(x_{1}+x_{2}\right)^{2}+2\left(\rho_{11}+\rho_{22}\right)\left(x_{1}+x_{2}\right)\left(y_{1}+y_{2}\right)+2\left(y_{1}+y_{2}\right)^{2}}}
\end{aligned}
$$




$$
\frac{x_{2}^{2}}{2}+\frac{y_{2}^{2}}{2}=\frac{\left(x_{1}+x_{2}\right)^{2}+\left(y_{1}+y_{2}\right)^{2}}{\sqrt{2\left(x_{1}+x_{2}\right)^{2}+2\left(\rho_{11}+\rho_{22}\right)\left(x_{1}+x_{2}\right)\left(y_{1}+y_{2}\right)+2\left(y_{1}+y_{2}\right)^{2}}}
$$

and

$$
\frac{x_{2}^{2}}{2}+\frac{y_{2}^{2}}{2}=\frac{\left(x_{2}-x_{1}\right)^{2}+\left(y_{1}-y_{2}\right)^{2}}{\sqrt{2\left(x_{2}-x_{1}\right)^{2}+2\left(\rho_{12}+\rho_{21}\right)\left(x_{2}-x_{1}\right)\left(y_{1}-y_{2}\right)+\left(y_{1}-y_{2}\right)^{2}}},
$$

with $0<x_{1}<x_{2}, 0<y_{1}<y_{2}$, and $-1, \rho_{i j}<1$, will suffice. Equation A.3 is actually entailed if both Equations A. 1 and A.2 are satisfied. These constraints can be expressed as a relationship between the correlation coefficients and the mean parameters that must hold,

$$
\left(\rho_{11}+\rho_{22}\right)=\frac{\left[\left(x_{1}+x_{2}\right)^{2}+\left(y_{1}+y_{2}\right)\right]^{2}-\left(x_{2}^{2}+y_{2}^{2}\right)\left(x_{1}+x_{2}\right)^{2}-\left(x_{2}^{2}+y_{2}^{2}\right)\left(y_{1}+y_{2}\right)^{2}}{\left(x_{2}^{2}+y_{2}^{2}\right)\left(x_{1}+x_{2}\right)\left(y_{1}+y_{2}\right)}
$$

and

$$
\left(\rho_{12}+\rho_{21}\right)=\frac{\left[\left(x_{2}-x_{1}\right)^{2}+\left(y_{1}+y_{2}\right)^{2}\right]^{2}-\left(x_{2}^{2}+y_{2}^{2}\right)\left(x_{2}-x_{1}\right)^{2}-\left(x_{2}^{2}+y_{2}^{2}\right)\left(y_{1}-y_{2}\right)^{2}}{\left(x_{2}^{2}+y_{2}^{2}\right)\left(x_{2}-x_{1}\right)\left(y_{1}-y_{2}\right)} .
$$

Possible mean parameter values for which the right-hand sides of Equations A.4 and A.5 lie in the open interval $(-2,2)$ provide the solutions. The reader can verify that if $x_{1}=1 / 4, x_{2}=2, y_{1}=1 / 4$, and $y_{2}=2$, this will give $\rho_{11}+\rho_{22}=17 / 32$ and $\rho_{12}+\rho_{21}=15 / 32$. For these values,

$$
\left(d_{A_{1} B_{1}, A_{2} B_{2}}^{\prime}\right)^{2}=\left(d_{A_{1} B_{1}, A_{2} B_{1}}^{\prime}\right)^{2}=4=\left(d_{A B_{j}}^{\prime}\right)^{2}+\left(d_{A_{i} B}^{\prime}\right)^{2} \text {. }
$$

PROOF b. A stimulus configuration satisfying perceptual separability but failing Condition iii is shown in Figure 4 with the parameters given in the text. It is straightforward to show that, for $\rho \neq 0$ (and $\neq \pm 1$ ), that Condition iii fails. This is because $d_{A_{1} B_{1}, A_{2} B_{2}}^{\prime}=1 / \sqrt{1+\rho}$ and $d_{A_{1} B_{2}, A_{2} B_{1}}^{\prime}=1 / \sqrt{1-\rho}$.

\section{Proof of Proposition B}

The ordinary formula for $d$ 's are used (i.e., taking the average of the variance in the denominator) rather than the Kadlec and Townsend version to facilitate comparison with standard signal detection theory.

NECESSITY. Without loss of generality, assume that the noise distribution is centered about $(0,0)$. If Conditions $\mathrm{i}$, ii, and iii hold, then the assumptions of the Tanner model are explicitly true, and $d_{1}^{\prime}=\mu_{1}^{x} / \sigma$ and $d_{2}^{\prime}=$ $\mu_{2}^{y} / \sigma$. Condition iv implies that $d_{1,2}^{\prime}$ can be found from Equation 1 (or 4 ). Let $\mathbf{U}_{i}$ be the univariate projection of the distribution for $S_{i}$ onto the line connecting

$$
\boldsymbol{\mu}_{N+S_{1}}=\left(\begin{array}{c}
\mu_{1}^{x} \\
0
\end{array}\right)
$$

and

$$
\boldsymbol{\mu}_{N+S_{2}}=\left(\begin{array}{c}
0 \\
\mu_{1}^{y}
\end{array}\right)
$$

Then

and

$$
E\left(U_{1}\right)=\frac{\left(\mu_{1}^{x}\right)^{2}}{\sqrt{\left(\mu_{1}^{x}\right)^{2}+\left(\mu_{1}^{y}\right)^{2}}}
$$

$$
E\left(U_{2}\right)=\frac{\left(\mu_{2}^{y}\right)^{2}}{\sqrt{\left(\mu_{1}^{x}\right)^{2}+\left(\mu_{2}^{y}\right)^{2}}}
$$

with, for $i=1,2$,

$$
\operatorname{Var}\left(U_{i}\right)=\frac{1}{\left(\mu_{1}^{x}\right)^{2}+\left(\mu_{1}^{y}\right)^{2}}\left(\begin{array}{ll}
\mu_{i}^{x} & \mu_{2}^{y}
\end{array}\right)\left(\begin{array}{cc}
\sigma^{2} & 0 \\
0 & \sigma^{2}
\end{array}\right)\left(\begin{array}{l}
\mu_{1}^{x} \\
\mu_{2}^{y}
\end{array}\right)
$$


Thus,

$$
d_{1,2}^{\prime}=\frac{\sqrt{\left(\mu_{1}^{x}\right)^{2}+\left(\mu_{2}^{y}\right)^{2}}}{\sigma} .
$$

Substituting the three $d^{\prime}$ s into Equation 5 gives $\theta=90^{\circ}$, as desired.

SUFFICIENCY. Suppose the Tanner detectability model holds with $\theta=90^{\circ}$. Thus, the $y$-axis in the GRT model is the line through $\boldsymbol{\mu}_{N}=(0,0)$ and $\boldsymbol{\mu}_{N+S_{2}}=\left(0, d_{2}^{\prime}\right)$, and the $x$-axis is through $\boldsymbol{\mu}_{N}=(0,0)$ and $\boldsymbol{\mu}_{N+S_{1}}=\left(d_{1,0}^{\prime}\right)$. The linear transformation, $L$, is simply the identity, so the resulting distributions are Gaussian in the GRT representation, and the covariance matrices are equal and satisfy the Tanner assumptions of equal variances across the dimensions and zero correlation (i.e., Conditions $\mathrm{i}$, ii, and iii hold). If the $d_{1,2}^{\prime}$ satisfies Equation 5 , then the response probabilities used to compute it come from a decision process that is equivalent to the distance classifier between $S_{1}$ and $S_{2}$.

\section{Proof of Proposition C}

The proof follows from the observation that the decision strategy generating $d_{g}^{\prime}$ is the distance classifier. The discriminant function can be written as

$$
\boldsymbol{D}=\mathbf{b}^{T} \mathbf{x},
$$

where $\mathbf{b}$ is a vector of discriminant coefficients and $\mathbf{x}$ is the random observation. The coefficients of $\mathbf{b}$ satisfy

$$
\mathbf{b}=\Sigma^{-1}\left(\boldsymbol{\mu}_{2}-\boldsymbol{\mu}_{1}\right)
$$

If the linear discriminant function, $\boldsymbol{D}$, is parallel to $\mathbf{v}=\left(\boldsymbol{\mu}_{2}-\boldsymbol{\mu}_{1}\right)$, then $\mathbf{b}=c \mathbf{v}$ for some scalar, $c \neq 0$. That is,

$$
\sum^{-1}\left(\boldsymbol{\mu}_{2}-\boldsymbol{\mu}_{1}\right)=c\left(\boldsymbol{\mu}_{2}-\boldsymbol{\mu}_{1}\right)
$$

or

$$
\begin{gathered}
\frac{1}{c}\left(\boldsymbol{\mu}_{2}-\boldsymbol{\mu}_{1}\right)=\sum\left(\boldsymbol{\mu}_{2}-\boldsymbol{\mu}_{1}\right) \\
\lambda\left(\boldsymbol{\mu}_{2}-\boldsymbol{\mu}_{1}\right)=\Sigma\left(\boldsymbol{\mu}_{2}-\boldsymbol{\mu}_{1}\right) .
\end{gathered}
$$

This implies that $\lambda(=1 / c)$ is an eigenvalue of $\sum$, and $\left(\boldsymbol{\mu}_{2}-\boldsymbol{\mu}_{1}\right)$ is the associated eigenvector. Recall, the Mahalanobis distance squared is

$$
\begin{aligned}
\Delta^{2}\left(P_{1}, P_{2}\right) & =\left(\boldsymbol{\mu}_{2}-\boldsymbol{\mu}_{1}\right)^{T} \sum^{-1}\left(\boldsymbol{\mu}_{2}-\boldsymbol{\mu}_{1}\right) \\
& =\left(\boldsymbol{\mu}_{2}-\boldsymbol{\mu}_{1}\right)^{T}\left[\frac{1}{\lambda}\left(\boldsymbol{\mu}_{2}-\boldsymbol{\mu}_{1}\right)\right] \\
& =\frac{1}{\lambda}\left(\boldsymbol{\mu}_{2}-\boldsymbol{\mu}_{1}\right)^{T}\left(\boldsymbol{\mu}_{2}-\boldsymbol{\mu}_{1}\right) .
\end{aligned}
$$

Now, from Equation 1, with $\Sigma_{1}=\Sigma_{2}=\sum$,

$$
\begin{aligned}
2\left(d_{g}\right)^{2} & =\frac{\left[\left(\boldsymbol{\mu}_{2}-\boldsymbol{\mu}_{1}\right)^{T}\left(\boldsymbol{\mu}_{2}-\boldsymbol{\mu}_{1}\right)\right]^{2}}{\left(\boldsymbol{\mu}_{2}-\boldsymbol{\mu}_{1}\right)^{T}\left[\lambda\left(\boldsymbol{\mu}_{2}-\boldsymbol{\mu}_{1}\right)\right]} \\
& =\left(\frac{1}{\lambda}\right)\left(\boldsymbol{\mu}_{2}-\boldsymbol{\mu}_{1}\right)^{T}\left(\boldsymbol{\mu}_{2}-\boldsymbol{\mu}_{1}\right),
\end{aligned}
$$

which proves the result.

The fact that $\left(\boldsymbol{\mu}_{2}-\boldsymbol{\mu}_{1}\right)$ is an eigenvector of $\sum$ means that, in the special case when the best linear discriminant is equivalent to the distance classifier, the decision axis lies along one of the (common) principal axes of the distributions (a situation depicted in Figure 4).

(Manuscript received June 2, 1997;

revision accepted for publication July 27, 1998. 